\title{
Urban-rural disparities in cardiovascular disease risks among middle-aged and older Chinese: two decades of urbanisation
}

\author{
Nan Zhang \\ Manchester Institute for Collaborative Research on Ageing (MICRA), Social Statistics, School of Social \\ Sciences, The University of Manchester, Manchester, UK \\ Corresponding author. Email: nan.zhang-2@manchester.ac.uk
}

(Accepted 12 December 2018; first published online 30 January 2019)

\begin{abstract}
China has been undergoing dramatic socio-economic and demographic changes in the last few decades. The rapid growth of the ageing population will pose tremendous challenges to its public health and social welfare system. This study aims to examine how urbanisation has impacted cardiovascular disease (CVD) risks among middle-aged and older Chinese in two decades, from 1991 to 2011. Data were drawn from a nationwide longitudinal data-set of the China Health and Nutrition Survey (CHNS) (sweeps 1991, 1993, 1997, 2000, 2004, 2006, 2009 and 2011). Participants aged $\geqslant 45$ years old were included. A dynamic urbanisation index was created for each community (village or neighbourhood) based on community-level data that can reveal the heterogeneity within and across places and capture dimensions of social, economic and physical characteristics of urban living over time and space. Multi-level modelling analyses (level 1: occasions; level 2: individuals; level 3: households; level 4: communities) were performed on outcomes of CVD risks such as body mass index, waist circumference, and systolic and diastolic blood pressure. The results show upward trends in all CVD risks for both genders over 20 years of urbanisation after adjustment for socio-economic and demographic confounders. Urbanisation in China is associated with absolute increases in CVD risks over time among its middle-aged and older people, despite its contribution to relative reduction of the rural-urban gap over time. This is particularly true for women from the least urbanised areas. It is relevant to inform policy-making processes to target the most vulnerable groups of older people in China during its rapid urbanisation process. There is a possibility for policy intervention to reduce inequality during the process of China's planned urbanisation.
\end{abstract}

Keywords: urbanisation; ageing; cardiovascular diease risks; inequality; China

\section{Introduction}

China is undergoing rapid urbanisation. It will reach the same levels of urbanisation much quicker and the number of people involved in this shift will be much larger

(c) Cambridge University Press 2019. This is an Open Access article, distributed under the terms of the Creative Commons Attribution licence (http://creativecommons.org/licenses/by/4.0/), which permits unrestricted re-use, distribution, and reproduction in any medium, provided the original work is properly cited. 
compared to other countries (McGee, 2008), with the proportion of the urban population rising from 18 per cent (172.45 million) in 1978 to 55 per cent (749.16 million) in 2014 (National Bureau of Statistics of China, 2015). Urbanisation is associated with a range of changes in environmental and lifestyle factors that may affect individual health and wellbeing. As the pace of urbanisation accelerates, new challenges arise to characterise these environments, and to understand their positive and negative implications for health (McDade and Adair, 2001). However, attributing diseases to specific environmental influences has proved elusive (Zhu et al., 2011). Factors beyond individual level, such as the social and physical environment, are the primary determinants of the health and wellbeing of people (Vlahov et al., 2005).

Three theoretical perspectives have been proposed regarding research on urban health. The urban penalty approach argues that cities concentrate poor people and expose them to unhealthy physical and social environments. Urban sprawl focuses on the adverse health and environmental impacts of urban growth into outlying areas. These two approaches stress the negative aspects of health, and fail to consider the strengths within metropolitan areas. The third approach, 'urban health advantage', considers the special resources and protective effects of cities and only focuses on the positive aspects of urban living. All three perspectives are relatively static, describing health within a particular stage of urban development rather than exploring the changing processes between and within cities and their surrounding areas (Freudenberg et al., 2005). The impact of urbanisation on health is likely two-edged. Urbanisation is often associated with economic growth and poverty reduction. There are the benefits of ready access to health care, sanitation and secure nutrition, whilst there are also the evils of overcrowding, pollution, social deprivation, crime and stress-related illness. Some argue that urbanisation worsens the health status of the population affected, especially when health infrastructure and social security are under-developed (Moore et al., 2003; Godfrey and Julien, 2005). Therefore, whether and to what extent urbanisation is associated with the health and wellbeing of its population is an empirical question.

Unlike other countries, China's rapid urbanisation is not merely driven by spontaneous rural-urban migration, but also partly planned by the government which has maintained a bias in favour of urban residents to preserve regime stability (Ma, 2002; Chan and Buckingham, 2008). The process of China's rapid urbanisation may cause the unequal distribution of socio-economic resources and life chances among its population, thus contributing to rural-urban health inequalities (Zhang and Treiman, 2013). Alongside rapid urbanisation, China is entering a stage of 'super ageing' (Joseph and Phillips, 1999). It is predicted that China will become the world's most aged society by 2030 , with its proportion of senior citizens aged 60 years or over rising from 15.2 per cent ( 209 million) in 2015 to 36.5 per cent (492 million) in 2050 (United Nations Department of Economic and Social Affairs, 2015). Massive internal migration (mostly taken up by the working-age population) and rapid urbanisation are likely to drive the ageing problem to be more challenging in rural than in urban China (Chen et al., 2015): by 2030, the proportion of people aged 60 years or over in rural and urban areas will be 21.8 and 14.8 per cent, respectively (Cai and Wang, 2005). Urbanisation and migration have enhanced the geographical separation between adult children and their elderly parents, restricting 
familial social support (Joseph and Phillips, 1999; Chen and Liu, 2009). This, together with the impact of urbanisation on socio-economic structures, is likely to have significant implications for the health and wellbeing of older people in China.

Cardiovascular disease (CVD) is one of the leading causes of death in China, which is more prevalent among older people (Moran et al., 2010). Rapid urbanisation and improvements in people's living standards have contributed to the increase of CVD events. An upward temporal trend in CVD risk factors (i.e. obesity, hypertension) has been observed among Chinese elderly (Yu et al., 2000; Gordon-Larsen et al., 2014; Wang et al., 2014) in the past few decades. Population ageing is estimated to bear two-thirds of the total disease burden in China by 2030 (Chatterji et al., 2008), which is likely to drain its health-care and social welfare system.

Despite well-documented rural-urban inequalities among older people in China, there are few attempts to examine how these relationships have changed during the process of China's urbanisation. Most studies examining the health consequences of urbanisation employ a dichotomous urban-rural classification of individuals living in urban versus rural environments. This approach ignores the heterogeneity of experience within and across urban and rural situations, and fails to consider the specific aspects of local environments that are causally related to health outcomes (McDade and Adair, 2001). Modern 'rural' areas are now experiencing factors traditionally associated with the urban environment which has blurred urban-rural distinctions (Hugo, 2017). This is particularly relevant due to substantial regional variations in economic development and public resources across China. Another pitfall of employing an urban-rural dichotomy is its inability to detect changes in urbanisation over time (Dahly and Adair, 2007). This makes it impossible to examine temporal trends and patterns of urbanisation on health outcomes among older people in China. Previous research has tended to rely on a crosssectional study design and has failed to examine the dynamics in the health of middle-aged and older people in a rapidly urbanising society. This study will examine how the rural-urban gap in CVD risks changes over China's rapid urbanisation process. This study aims to answer two questions:

(1) What is the trend in CVD risks among Chinese middle-aged and older people over the last two decades?

(2) Do the trends in CVD risks change according to the degree of urbanisation?

\section{Methods}

\section{Study design and participants}

Data were drawn from the CHNS, an on-going open-cohort study that employs a multi-stage, random-clustered sampling process to draw a sample of about 4,400 households with a total of about 19,000 participants from over 200 communities or neighbourhoods in nine provinces, with the first round conducted in 1989. The CHNS covers nine provinces that vary substantially in geography, economic development, public resources and health indicators. The design, sampling and response rates are reported elsewhere (Popkin et al., 2009). The sample began with eight provinces (Liaoning, Jiangsu, Shandong, Henan, Hubei, Hunan, 
Guangxi and Guizhou) and added a ninth, Heilongjiang, in 1997, and three autonomous cities, Beijing, Shanghai and Chongqing, in 2011. The nine provinces were included and the three autonomous cities in 2011 were excluded from the analyses. This study used data from 1991, 1993, 1997, 2000, 2004, 2006, 2009 and 2011. In 1989, health data were only collected from pre-schoolers and adults aged 20-45 and therefore were not included in this study. Middle-aged and elder people $\geqslant 45$ years were included and underweight people $(\mathrm{N}=2,379)$ were excluded from analyses.

The institutional review committees from the University of North Carolina at Chapel Hill and the National Institute for Nutrition and Food Safety, China Centre for Disease Control and Prevention approved the survey protocols and instruments and the process for obtaining informed consent for the survey. All participants and/or their parents/guardians provided written informed consents for their participation in the survey.

\section{Outcomes}

CVD risks included body mass index (BMI), waist circumference (WC), systolic blood pressure (SBP) and diastolic blood pressure (DBP).

Anthropometric data were recorded for each individual within the household, as measured by health professionals. Height was measured without shoes to the nearest 0.1 centimetres $(\mathrm{cm})$ using a portable SECA stadiometer; weight was measured without shoes and in light clothing to the nearest 0.1 kilogram $(\mathrm{kg})$ on a calibrated beam balance. BMI $\left(\mathrm{kg} /\right.$ metre $\left.(\mathrm{m})^{2}\right)$ was calculated as weight $(\mathrm{kg})$ divided by height squared $\left(\mathrm{m}^{2}\right)$. Obesity was defined as BMI $\geqslant 28$ for men and women (Zhou, 2002). WC $(\mathrm{cm})$ was measured using an non-elastic tape at a point midway between the lowest rib margin and the iliac crest in a horizontal plane (Tuan et al., 2010). Abdominal obesity was defined as $\mathrm{WC} \geqslant 90 \mathrm{~cm}$ for men and $\mathrm{WC} \geqslant 80 \mathrm{~cm}$ for women (World Health Organization, 1997).

SBP and DBP were measured on the right arm, using mercury sphygmomanometers with appropriate cuff sizes (Blumenthal et al., 1977). Measures were collected in triplicate after a ten-minute seated rest and the mean of the three measurements was used in analyses. Hypertension was defined as an average $\mathrm{SBP} \geqslant 140$ millimetres of mercury $(\mathrm{mmHg})$ and/or an average $\mathrm{DBP} \geqslant 90 \mathrm{mmHg}$ (Gu et al., 2002).

\section{Exposure}

A dynamic measure, the urbanisation index, was created based on in-depth community contextual measures that capture major dimensions of modernisation across all 288 communities currently in the CHNS sample. The standardised, validated measure captures the changes in 12 dimensions: population density, economic activity, traditional markets, modern markets, transportation infrastructure, sanitation, communications, housing, education, diversity, health infrastructure and social services (Jones-Smith and Popkin, 2010). Each domain was scored from 0 to 10 , weighted equally and then added together scoring up to 120 . Each is based on numerous measures relevant to each dimension (Zhang 
et al., 2014) and can distinguish features of urban-rural places. This measure has been validated for content validity, reliability $(\alpha \geqslant 0.85)$ and stability $(r \geqslant 0.90)$ (Jones-Smith and Popkin, 2010). Also, a fixed measure, Hukou, which officially segregates China's population into 'urban residents' born in urban areas and 'rural residents or peasants' born in rural areas and is an important structural driver of inequality, was also examined.

\section{Covariates}

Covariates included age (centred at 45 years), quadratic term of age, education (completed years of formal education in regular school), log transformation of household income per capita (inflated to 2011) and survey years (1991, 1993, 1997, 2000, 2004, 2006, 2009 and 2011).

\section{Statistical analysis}

To account for the hierarchical nature of the CHNS, where multiple occasions (level 1) are nested within individuals (level 2) within households (level 3) clustered in villages/communities (level 4), multi-level modelling analyses were constructed within Stata/SE 13 (StataCorp, 2013) to correct for non-independence of observations due to geographic clustering and repeated observations of individuals. A multi-level approach also allows examination of neighbourhood/contextual effects given the key exposure - urbanisation - lies at the community level. The log likelihood ratio test was used to determine the preferred model among nested models for continuous outcomes including BMI, WC, SBP and DBP and the Wald test was used to test statistical significance in multi-level logistic regressions for categorical outcomes including obesity, abdominal obesity and hypertension. The better fit of the models is associated with lower values in -2 log likelihood statistics. Nested models are compared through deviance statistics (difference in $-2 \log$ likelihood) over the difference in degrees of freedom using an ordinary chi-square distribution. A significant difference between two nested models indicates that the models with the lowest value have a better fit to the data. Given gender differences in potential CVD risk factors and traditional gender roles in China, all analyses were stratified by sex.

To examine if there are any significant trends in outcomes, first a quadratic function of age and survey years was fitted (Model 1). This was followed by adjustment for urbanisation (Hukou or urbanisation index) to see if urbanisation has an independent impact on outcomes (Model 2). In order to examine if urbanisation impacts change over time, adjustment was made for the interaction between survey year and urbanisation (Model 3). All models were adjusted for family socioeconomic circumstances in terms of education and household income per capita to remove potential confounding effects. Also examined were the relationships between non-linear terms of the urbanisation index and outcomes, and non-linear terms were not significant (not reported here).

To enable an easier interpretation, time trends were plotted so that they can be interpreted visually. This was done by calculating predicted values of selected outcomes (obesity, abdominal obesity and hypertension) under different conditions of 
urbanisation, including the most urbanised areas (the top 20th percentile) and the least urbanised areas (the bottom 20th percentile), and showing the predicted relationship between outcome variables and survey year at different levels of urbanisation. These plots can offer an overall picture of trends in urban-rural inequalities in bio-markers among Chinese middle-aged and elder people over two decades.

\section{Results}

Table 1 summarises the socio-economic characteristics and CVD risks of middle-aged and older people aged $\geqslant 45$ across gender and survey years: the CHNS. The two decades from 1991 to 2011 saw persistent improvements in socioeconomic status (education and household income per capita) for both men and women regardless of their Hukou status. A clear rural-urban gap was observed for both men and women: urban people tend to fare better in terms of socioeconomic circumstances and to be more prone to CVD risks compared with their rural counterparts. Obesity, abdominal obesity in particular, increased dramatically among rural people, especially among women. Blood pressure and hypertension appeared to increase among rural people and did not show a consistent pattern among their urban peers.

Tables 2-4 present trends in CVD risks among middle-aged and older Chinese $\geqslant 45$ years during China's urbanisation process. The positive time trends were observed on all CVD risks for both genders after adjustment for socio-economic confounders (Model 1), and persisted regardless of urbanisation levels (Model 2). Higher levels of urbanisation appear to be associated with an increased risk of CVD and such impacts seem to be independent of individual socio-economic positions (Model 2). Significant negative interaction effects (between the urbanisation index and survey years) were observed on all CVD risks for both genders: the positive time trends tend to decrease as the areas become more urbanised (Model 3).

Figures 1 and 2 plot the time trends of the probability of obesity for the most urbanised and the least urbanised areas for males and females aged $\geqslant 45$ years. For both overall obesity and abdominal obesity, an upward trend was observed for both men and women regardless of levels of urbanisation. For males, those from the most urbanised areas appear to be more likely to be obese than those from the least urbanised areas. The gap between the two groups seems stable over two decades. However, the patterns appear to be more complex for females than for males. The risk of female obesity increased rapidly in particular for those from the least urbanised areas over time. The gap between two groups appears to shrink over two decades. This may suggest rapid urbanisation in China may help reduce inequality in obesity among women but may have limited impact on men.

Figure 3 illustrates the time trends of the probability of hypertension for men and women from the most urbanised and the least urbanised areas separately. The disparities between two urbanised groups appear to decrease and further cease: the probability of hypertension for those from the highest urbanised areas appears to decline while that of their least urbanised peers continues to increase. For women, the gap continued to converge till 2008 and after that the gap widened, whereas the least urbanised areas seem to override the most urbanised areas. This 
Table 1. General characteristics of middle-aged and older Chinese $\geqslant 45$ years by gender over time, China Health and Nutrition Survey $1991-2011$

\begin{tabular}{|c|c|c|c|c|c|c|c|c|}
\hline Variables & 1991 & 1993 & 1997 & 2000 & 2004 & 2006 & 2009 & 2011 \\
\hline & \multicolumn{8}{|c|}{ Mean values (standard deviations) or percentages } \\
\hline \multicolumn{9}{|l|}{ Male: urban: } \\
\hline $\mathrm{N}$ & 544 & 694 & 922 & 1,108 & 1,217 & 1,244 & 1,447 & 1,423 \\
\hline Age (years) & $59.33(8.72)$ & $59.57(9.21)$ & $60.03(10.16)$ & $59.61(10.51)$ & $60.65(10.63)$ & $60.51(10.63)$ & $60.69(10.85)$ & $61.02(11.07)$ \\
\hline Index & $62.47(9.16)$ & $61.97(11.26)$ & $68.07(11.78)$ & 74.35 (12.09) & $80.37(13.05)$ & $81.41(13.50)$ & $83.78(13.08)$ & $84.16(12.66)$ \\
\hline Education (years) & $6.34(4.95)$ & $6.68(4.72)$ & $7.09(4.46)$ & $8.04(4.42)$ & $8.84(4.10)$ & $9.27(4.19)$ & $9.36(4.0)$ & $9.51(3.82)$ \\
\hline $\begin{array}{l}\text { Log household income per } \\
\text { capita }\end{array}$ & $8.17(0.58)$ & $8.16(0.80)$ & $8.27(0.97)$ & $8.56(1.17)$ & $8.86(1.29)$ & $8.90(1.41)$ & $9.26(1.46)$ & $9.46(1.28)$ \\
\hline Height $(\mathrm{cm})$ & $165.10(5.99)$ & $165.06(5.91)$ & $165.81(5.99)$ & $166.23(6.13)$ & $166.29(6.48)$ & $166.69(6.37)$ & $167.31(6.43)$ & $167.14(6.43)$ \\
\hline Weight (kg) & $63.84(9.72)$ & $63.53(9.58)$ & $65.76(10.29)$ & $66.39(10.15)$ & $67.11(10.14)$ & $67.21(9.97)$ & $68.47(10.51)$ & $69.14(12.17)$ \\
\hline BMI $\left(\mathrm{kg} / \mathrm{m}^{2}\right)$ & $23.37(3.04)$ & $23.28(3.06)$ & $23.89(3.20)$ & $24.0(3.19)$ & $24.21(3.07)$ & $24.16(3.07)$ & $24.41(3.24)$ & $24.71(3.87)$ \\
\hline Waist circumference $(\mathrm{cm})$ & - & $82.98(9.73)$ & $84.64(10.51)$ & $85.82(9.91)$ & $86.62(9.51)$ & $86.91(9.51)$ & $88.18(9.55)$ & $88.50(10.19)$ \\
\hline $\begin{array}{l}\text { Systolic blood pressure } \\
(\mathrm{mmHg})\end{array}$ & $131.67(22.49)$ & $128.74(21.10)$ & $131.12(20.05)$ & $129.56(18.51)$ & $131.77(18.91)$ & $128.85(17.88)$ & $132.35(18.31)$ & $130.34(17.37)$ \\
\hline $\begin{array}{l}\text { Diastolic blood pressure } \\
(\mathrm{mmHg})\end{array}$ & $82.55(12.30)$ & $82.26(13.23)$ & $84.04(11.39)$ & $83.20(11.07)$ & $82.94(10.98)$ & $82.49(10.81)$ & $84.44(11.27)$ & $82.83(10.84)$ \\
\hline Obesity (\%) & 9.36 & 7.89 & 11.86 & 11.48 & 10.94 & 11.05 & 12.30 & 12.91 \\
\hline Abdominal obesity (\%) & - & 26.38 & 32.79 & 36.44 & 37.23 & 39.94 & 43.30 & 46.32 \\
\hline Hypertension (\%) & 41.63 & 41.52 & 48.26 & 41.48 & 46.02 & 36.94 & 49.76 & 40.17 \\
\hline
\end{tabular}


Table 1. (Continued.)

\begin{tabular}{|c|c|c|c|c|c|c|c|c|}
\hline Variables & 1991 & 1993 & 1997 & 2000 & 2004 & 2006 & 2009 & 2011 \\
\hline \multicolumn{9}{|l|}{ Male: rural: } \\
\hline $\mathrm{N}$ & 805 & 828 & 1,104 & 1,358 & 1,506 & 1,600 & 1,787 & 1,896 \\
\hline Age (years) & $55.67(8.64)$ & $56.37(9.24)$ & $57.11(9.97)$ & 57.09 (9.69) & 57.77 (9.54) & $58.66(9.37)$ & $59.04(10.07)$ & $59.32(10.04)$ \\
\hline Index & $36.84(12.07)$ & 37.45 (11.54) & $41.58(13.08)$ & $47.84(13.35)$ & $49.11(14.23)$ & $52.24(15.40)$ & 55.54 (13.95) & $58.05(14.40)$ \\
\hline Education (years) & $3.81(3.41)$ & $4.08(3.39)$ & $4.62(3.34)$ & $5.39(3.25)$ & $6.43(3.28)$ & $6.14(3.73)$ & $6.51(3.51)$ & $6.81(3.43)$ \\
\hline $\begin{array}{l}\text { Log household income per } \\
\text { capita }\end{array}$ & $7.57(0.96)$ & $7.56(1.17)$ & $7.73(1.43)$ & $7.88(1.36)$ & $8.02(1.53)$ & $8.13(1.50)$ & $8.55(1.58)$ & $8.74(1.46)$ \\
\hline Height $(\mathrm{cm})$ & $162.39(6.17)$ & $162.51(6.20)$ & $163.12(6.14)$ & $164.16(6.10)$ & $164.21(6.33)$ & $164.31(6.43)$ & $164.93(6.43)$ & $164.67(7.99)$ \\
\hline Weight (kg) & $56.81(7.45)$ & $57.27(7.60)$ & $58.86(8.66)$ & $60.80(9.20)$ & $61.18(9.37)$ & $61.91(9.69)$ & $63.29(10.02)$ & $64.53(12.56)$ \\
\hline BMI $\left(\mathrm{kg} / \mathrm{m}^{2}\right)$ & $21.50(2.10)$ & $21.63(2.09)$ & $22.06(2.52)$ & $22.51(2.66)$ & $22.62(2.67)$ & $22.85(2.76)$ & $23.18(2.86)$ & $23.85(6.20)$ \\
\hline Waist circumference $(\mathrm{cm})$ & - & $76.71(7.43)$ & $78.51(8.25)$ & $80.76(8.67)$ & $81.78(8.82)$ & $82.10(8.88)$ & $84.17(9.40)$ & $85.18(9.88)$ \\
\hline $\begin{array}{l}\text { Systolic blood pressure } \\
(\mathrm{mmHg})\end{array}$ & $120.05(18.50)$ & $121.21(17.58)$ & $123.13(17.79)$ & $125.76(18.19)$ & $125.91(18.56)$ & $126.35(18.16)$ & $129.21(18.21)$ & $129.23(18.20)$ \\
\hline $\begin{array}{l}\text { Diastolic blood pressure } \\
\text { (mmHg) }\end{array}$ & $77.74(12.17)$ & $78.99(10.94)$ & $79.64(11.71)$ & 80.88 (11.59) & $81.06(12.07)$ & $81.49(11.60)$ & $83.58(11.51)$ & $82.63(11.43)$ \\
\hline Obesity (\%) & 1.65 & 0.85 & 2.85 & 3.60 & 4.00 & 4.11 & 5.58 & 8.97 \\
\hline Abdominal obesity (\%) & - & 5.85 & 12.0 & 16.26 & 19.22 & 20.83 & 27.61 & 32.18 \\
\hline Hypertension (\%) & 26.52 & 27.98 & 29.93 & 33.49 & 34.25 & 35.63 & 46.23 & 36.95 \\
\hline \multicolumn{9}{|l|}{ Female: urban: } \\
\hline $\mathrm{N}$ & 595 & 723 & 912 & 1,100 & 1,247 & 1,295 & 1,452 & 1,410 \\
\hline Age (years) & $59.73(9.18)$ & $60.51(9.87)$ & $61.01(10.50)$ & $59.99(10.75)$ & $60.47(10.77)$ & $61.33(10.97)$ & $61.12(10.91)$ & $61.38(11.05)$ \\
\hline
\end{tabular}




\begin{tabular}{|c|c|c|c|c|c|c|c|c|}
\hline Index & $62.97(7.26)$ & $64.04(8.53)$ & $69.72(9.60)$ & $76.30(9.50)$ & $81.88(11.24)$ & $82.93(11.88)$ & $85.14(11.48)$ & $85.30(11.19)$ \\
\hline Education (years) & $3.17(4.14)$ & $3.50(4.18)$ & $4.15(4.25)$ & $5.41(4.58)$ & $6.44(4.57)$ & $6.68(4.83)$ & $7.12(4.54)$ & $7.61(4.49)$ \\
\hline $\begin{array}{l}\text { Log household income per } \\
\text { capita }\end{array}$ & $8.09(0.70)$ & $8.08(0.91)$ & $8.22(0.99)$ & $8.50(1.19)$ & $8.81(1.29)$ & $8.85(1.44)$ & $9.22(1.47)$ & $9.36(1.47)$ \\
\hline Height (cm) & $153.38(6.16)$ & $153.64(6.10)$ & $154.01(5.78)$ & $155.18(6.02)$ & $155.08(6.20)$ & $155.34(6.81)$ & $156.26(6.29)$ & $155.82(6.91)$ \\
\hline Weight (kg) & $56.70(9.44)$ & $56.44(9.11)$ & $57.96(9.55)$ & $59.13(9.21)$ & $59.09(9.37)$ & $59.19(9.13)$ & $59.78(9.16)$ & $60.06(11.23)$ \\
\hline BMI $\left(\mathrm{kg} / \mathrm{m}^{2}\right)$ & $24.06(3.43)$ & $23.88(3.28)$ & $24.39(3.42)$ & $24.52(3.38)$ & $24.55(3.45)$ & $24.58(4.62)$ & $24.46(3.27)$ & $24.73(4.39)$ \\
\hline Waist circumference $(\mathrm{cm})$ & - & $82.53(10.41)$ & $82.42(10.39)$ & $83.68(10.06)$ & $83.83(10.01)$ & 84.05 (9.49) & $84.58(9.60)$ & $84.49(10.28)$ \\
\hline $\begin{array}{l}\text { Systolic blood pressure } \\
(\mathrm{mmHg})\end{array}$ & $128.71(23.52)$ & $128.66(23.82)$ & $130.85(22.87)$ & $129.07(20.92)$ & $130.50(21.13)$ & $127.61(19.75)$ & $130.58(19.74)$ & $128.14(18.62)$ \\
\hline $\begin{array}{l}\text { Diastolic blood pressure } \\
(\mathrm{mmHg})\end{array}$ & $80.61(12.31)$ & 80.68 (12.99) & $82.59(12.40)$ & $81.62(12.43)$ & $80.89(12.01)$ & $80.47(11.10)$ & $81.95(10.64)$ & $79.70(10.75)$ \\
\hline Obesity (\%) & 13.53 & 11.11 & 13.70 & 14.23 & 15.61 & 14.47 & 14.40 & 15.05 \\
\hline Abdominal obesity (\%) & - & 62.78 & 60.83 & 64.75 & 65.69 & 66.96 & 68.61 & 69.08 \\
\hline Hypertension (\%) & 37.17 & 41.63 & 45.32 & 36.08 & 40.38 & 32.81 & 45.95 & 32.02 \\
\hline \multicolumn{9}{|l|}{ Female: rural: } \\
\hline $\mathrm{N}$ & 818 & 861 & 1,152 & 1,457 & 1,605 & 1,738 & 1,948 & 2,049 \\
\hline Age (years) & $57.51(10.0)$ & $57.93(10.53)$ & $58.28(11.22)$ & $58.11(10.94)$ & $58.81(10.71)$ & $59.72(10.66)$ & $59.92(11.08)$ & $602.9(10.98)$ \\
\hline Index & $37.0(12.17)$ & $37.76(11.93)$ & $42.51(13.95)$ & 48.09 (13.59) & $50.16(14.47)$ & $52.95(15.51)$ & $56.10(14.22)$ & $58.86(15.0)$ \\
\hline Education (years) & $1.31(2.53)$ & $1.54(2.60)$ & $2.02(2.87)$ & $2.62(3.16)$ & $3.48(3.51)$ & $3.35(3.69)$ & $3.84(3.79)$ & $4.25(3.86)$ \\
\hline $\begin{array}{l}\text { Log household income per } \\
\text { capita }\end{array}$ & $7.53(1.04)$ & $7.58(1.21)$ & $7.73(1.52)$ & $7.89(1.38)$ & $8.07(1.43)$ & $8.13(1.46)$ & $8.56(1.59)$ & $8.71(1.50)$ \\
\hline Height $(\mathrm{cm})$ & $151.21(6.15)$ & $151.61(5.92)$ & $152.37(6.01)$ & $152.91(6.04)$ & $153.06(6.47)$ & $153.33(6.46)$ & $153.77(6.49)$ & $153.75(6.82)$ \\
\hline Weight (kg) & $50.71(7.86)$ & $51.49(7.98)$ & $53.09(8.56)$ & $54.66(8.72)$ & $55.56(9.17)$ & $56.12(9.35)$ & $56.90(9.45)$ & $57.83(10.55)$ \\
\hline
\end{tabular}


Table 1. (Continued.)

\begin{tabular}{lcccccccc}
\hline Variables & 1991 & 1993 & 1997 & 2000 & 2004 & 2006 & 2009 & 2011 \\
\hline BMI (kg/m $\left.{ }^{2}\right)$ & $22.13(2.80)$ & $22.37(2.87)$ & $22.82(3.09)$ & $23.32(3.06)$ & $23.65(3.18)$ & $23.79(3.20)$ & $24.0(3.29)$ & $24.42(4.14)$ \\
\hline Waist circumference $(\mathrm{cm})$ & - & $77.23(8.76)$ & $78.44(9.07)$ & $80.56(9.20)$ & $81.63(9.32)$ & $82.29(9.74)$ & $84.01(9.65)$ & $84.83(10.59)$ \\
\hline $\begin{array}{l}\text { Systolic blood pressure } \\
\text { (mmHg) }\end{array}$ & $119.10(20.56)$ & $120.38(20.26)$ & $124.24(21.09)$ & $124.37(21.16)$ & $124.98(21.75)$ & $126.01(20.20)$ & $130.04(21.09)$ & $129.83(20.83)$ \\
$\begin{array}{l}\text { Diastolic blood pressure } \\
\text { (mmHg) }\end{array}$ & $76.12(12.05)$ & $77.67(12.61)$ & $78.55(11.69)$ & $79.15(12.24)$ & $79.33(13.18)$ & $79.95(11.62)$ & $82.06(12.04)$ & $81.12(12.07)$ \\
\hline \begin{tabular}{l} 
Obesity (\%) \\
\hline Abdominal obesity (\%)
\end{tabular} & 3.37 & 4.23 & 6.26 & 7.60 & 9.51 & 11.28 & 12.47 & 14.70 \\
\hline Hypertension (\%) & - & 36.86 & 42.16 & 52.34 & 57.78 & 59.51 & 66.15 & 67.32 \\
\hline
\end{tabular}

Notes: Index: urbanisation index. cm: centimetre. kg: kilogram. m: metre. BMI: body mass index. mmHg: millimetres of mercury. 


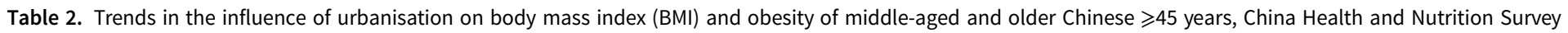
1991-2011

\begin{tabular}{|c|c|c|c|c|c|c|}
\hline & \multicolumn{2}{|c|}{ Model 1} & \multicolumn{2}{|c|}{ Model 2} & \multicolumn{2}{|c|}{ Model 3} \\
\hline & Coefficient & $95 \% \mathrm{Cl}$ & Coefficient & $95 \% \mathrm{Cl}$ & Coefficient & $95 \% \mathrm{Cl}$ \\
\hline \multicolumn{7}{|l|}{ BMI: } \\
\hline \multicolumn{7}{|l|}{ Male: } \\
\hline Year & $0.082^{\star \star \star}$ & $0.073,0.091$ & $0.066^{\star \star \star}$ & $0.055,0.076$ & $0.092^{\star \star \star}$ & $0.069,0.115$ \\
\hline Index & & & $0.015^{\star \star \star}$ & $0.010,0.021$ & $0.022^{\star \star \star}$ & $0.014,0.029$ \\
\hline Year $\times$ Index & & & & & $-0.0005^{\star}$ & $-0.0008,-0.0001$ \\
\hline \multicolumn{7}{|l|}{ Female: } \\
\hline Year & $0.078^{\star \star \star}$ & $0.069,0.087$ & $0.069^{\star \star \star}$ & $0.059,0.079$ & $0.127^{\star \star \star}$ & $0.107,0.147$ \\
\hline Index & & & $0.009 \star \star \star$ & $0.004,0.013$ & $0.023^{\star \star \star}$ & $0.016,0.029$ \\
\hline Year $\times$ Index & & & & & $-0.001^{\star \star \star}$ & $-0.001,-0.0007$ \\
\hline \multicolumn{7}{|l|}{ Obesity: } \\
\hline \multicolumn{7}{|l|}{ Male: } \\
\hline Year & $0.039^{\star \star \star}$ & $0.027,0.051$ & $0.019^{\star \star}$ & $0.038^{\star \star \star}$ & $0.132^{\star \star \star}$ & $0.085,0.179$ \\
\hline Index & & & $0.019^{\star \star \star}$ & $0.008^{\star \star}$ & $0.044^{\star \star \star}$ & $0.032,0.057$ \\
\hline Year $\times$ Index & & & & & $-0.002^{\star \star \star}$ & $-0.002,-0.001$ \\
\hline \multicolumn{7}{|l|}{ Female: } \\
\hline Year & $0.046^{\star \star \star}$ & $0.037,0.055$ & $0.038^{\star \star \star}$ & $0.027,0.049$ & $0.105^{\star \star \star}$ & $0.074,0.136$ \\
\hline Index & & & $0.008^{\star \star}$ & $0.002,0.013$ & $0.024^{\star \star \star}$ & $0.015,0.032$ \\
\hline Year $\times$ Index & & & & & $-0.001^{\star \star}$ & $-0.002,-0.001$ \\
\hline
\end{tabular}

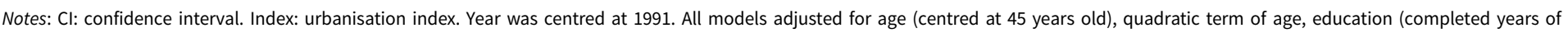
formal education in regular school) and log transformation of household income per capita.

Significance levels: ${ }^{\star} p<0.05,{ }^{\star \star} p<0.01,{ }^{\star \star \star} p<0.001$. 
Table 3. Trends in the influence of urbanisation on waist circumference and abdominal obesity of middle-aged and older Chinese $\geqslant 45$ years, China Health and Nutrition Survey 1991-2011

\begin{tabular}{|c|c|c|c|c|c|c|}
\hline & \multicolumn{2}{|c|}{ Model 1} & \multicolumn{2}{|c|}{ Model 2} & \multicolumn{2}{|c|}{ Model 3} \\
\hline & Coefficient & $95 \% \mathrm{Cl}$ & Coefficient & $95 \% \mathrm{Cl}$ & Coefficient & $95 \% \mathrm{Cl}$ \\
\hline \multicolumn{7}{|c|}{ Waist circumference: } \\
\hline \multicolumn{7}{|l|}{ Male: } \\
\hline Year & $0.357^{\star \star \star}$ & $0.330,0.384$ & $0.309^{\star \star \star}$ & $0.278,0.340$ & $0.466^{\star \star \star}$ & $0.397,0.536$ \\
\hline Index & & & $0.046^{\star \star \star}$ & $0.031,0.061$ & $0.083^{\star \star \star}$ & $0.062,0.104$ \\
\hline Year $\times$ Index & & & & & $-0.003^{\star * \star}$ & $-0.004,-0.002$ \\
\hline \multicolumn{7}{|l|}{ Female: } \\
\hline Year & $0.308^{\star \star \star}$ & $0.280,0.335$ & $0.271^{\star \star \star}$ & $0.240,0.303$ & $0.520^{\star \star \star}$ & $0.450,0.591$ \\
\hline Index & & & $0.037^{\star \star \star}$ & $0.022,0.052$ & $0.097^{\star \star \star}$ & $0.076,0.118$ \\
\hline Year $\times$ Index & & & & & $-0.004^{\star \star \star}$ & $-0.005,-0.003$ \\
\hline \multicolumn{7}{|c|}{ Abdominal obesity: } \\
\hline \multicolumn{7}{|l|}{ Male: } \\
\hline Year & $0.062^{\star \star \star}$ & $0.053,0.070$ & $0.048^{\star \star \star}$ & $0.038,0.058$ & $0.141^{\star \star \star}$ & $0.111,0.171$ \\
\hline Index & & & $0.013^{\star \star \star}$ & $0.008,0.018$ & $0.035^{\star \star \star}$ & $0.027,0.043$ \\
\hline Year $\times$ Index & & & & & $-0.001^{\star \star \star}$ & $-0.002,-0.001$ \\
\hline \multicolumn{7}{|l|}{ Female: } \\
\hline Year & $0.050^{\star \star \star}$ & $0.044,0.057$ & $0.044^{\star \star \star}$ & $0.037,0.052$ & $0.121^{\star \star \star}$ & $0.100,0.142$ \\
\hline Index & & & $0.006^{\star \star}$ & $0.002,0.010$ & $0.023^{\star \star \star}$ & $0.018,0.030$ \\
\hline Year $\times$ Index & & & & & $-0.001^{\star \star \star}$ & $-0.002,-0.001$ \\
\hline
\end{tabular}

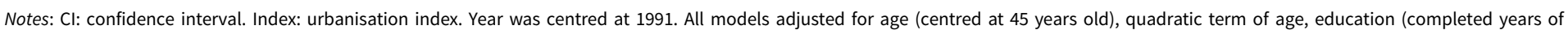
formal education in regular school) and log transformation of household income per capita.

Significance levels: ${ }^{\star \star} p<0.01,{ }^{\star \star \star} p<0.001$. 
Table 4. Trends in the influence of urbanisation on systolic blood pressure, diastolic blood pressure and hypertension of middle-aged and older Chinese $\geqslant 45$ years, China Health and Nutrition Survey 1991-2011

\begin{tabular}{|c|c|c|c|c|c|c|}
\hline & \multicolumn{2}{|c|}{ Model 1} & \multicolumn{2}{|c|}{ Model 2} & \multicolumn{2}{|c|}{ Model 3} \\
\hline & Coefficient & $95 \% \mathrm{Cl}$ & Coefficient & $95 \% \mathrm{Cl}$ & Coefficient & $95 \% \mathrm{Cl}$ \\
\hline \multicolumn{7}{|c|}{ Systolic blood pressure: } \\
\hline \multicolumn{7}{|l|}{ Male: } \\
\hline Year & $0.170^{\star \star \star}$ & $0.121,0.220$ & $0.107^{\star \star \star}$ & $0.051,0.162$ & $0.623^{\star \star \star}$ & $0.488,0.759$ \\
\hline Index & & & $0.064^{\star \star \star}$ & $0.038,0.091$ & $0.186^{\star \star \star}$ & $0.147,0.226$ \\
\hline Year $\times$ Index & & & & & $-0.009^{\star \star \star}$ & $-0.011,-0.007$ \\
\hline \multicolumn{7}{|l|}{ Female: } \\
\hline Year & $0.212^{\star \star \star}$ & $0.158,0.265$ & $0.185^{\star \star \star}$ & $0.125,0.246$ & $0.748^{\star \star \star}$ & $0.606,0.890$ \\
\hline Index & & & 0.029 & $-0.001,0.059$ & $0.162^{\star \star \star}$ & $0.120,0.205$ \\
\hline Year $\times$ Index & & & & & $-0.010^{\star \star \star}$ & $-0.012,-0.008$ \\
\hline \multicolumn{7}{|c|}{ Diastolic blood pressure: } \\
\hline \multicolumn{7}{|l|}{ Male: } \\
\hline Year & $0.117^{\star \star \star}$ & $0.086,0.149$ & $0.097^{\star \star \star}$ & $0.061,0.134$ & $0.402^{\star \star \star}$ & $0.314,0.489$ \\
\hline Index & & & $0.020^{*}$ & $0.002,0.038$ & $0.092^{\star \star \star}$ & $0.066,0.118$ \\
\hline Year $\times$ Index & & & & & $-0.005^{\star \star \star}$ & $-0.007,-0.004$ \\
\hline \multicolumn{7}{|l|}{ Female: } \\
\hline Year & $0.091^{\star \star \star}$ & $0.059,0.122$ & $0.084^{\star \star \star}$ & $0.048,0.121$ & $0.408^{\star \star \star}$ & $0.324,0.492$ \\
\hline Index & & & 0.008 & $-0.011,0.026$ & $0.085^{\star \star \star}$ & $0.059,0.111$ \\
\hline Year $\times$ Index & & & & & $-0.006^{\star \star \star}$ & $-0.007,-0.004$ \\
\hline
\end{tabular}


Table 4. (Continued.)

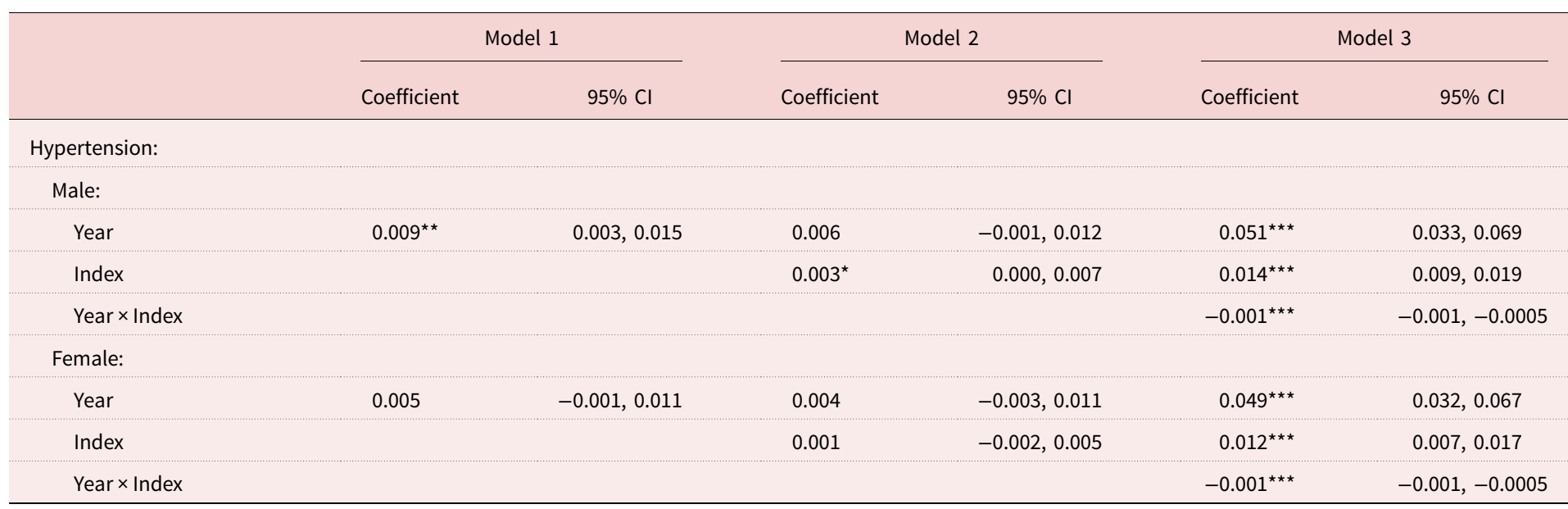

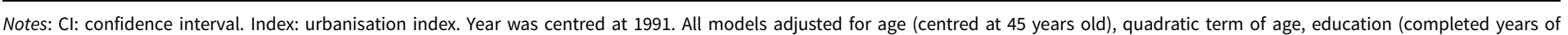
formal education in regular school) and log transformation of household income per capita.

Significance levels: ${ }^{*} p<0.05,{ }^{\star \star} p<0.01,{ }^{\star * \star} p<0.001$. 
(a)

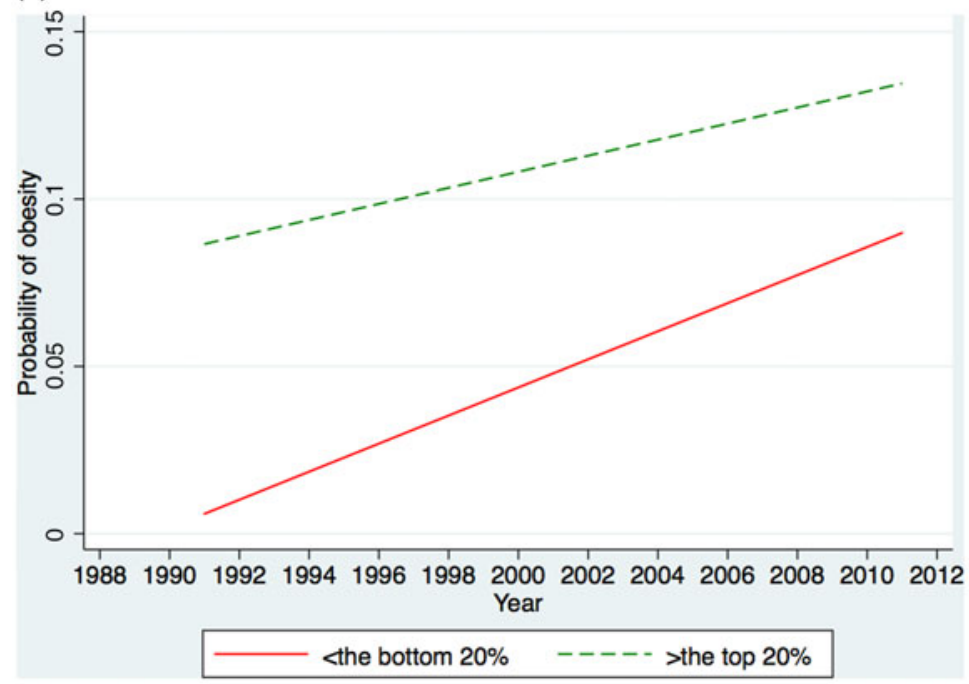

(b)

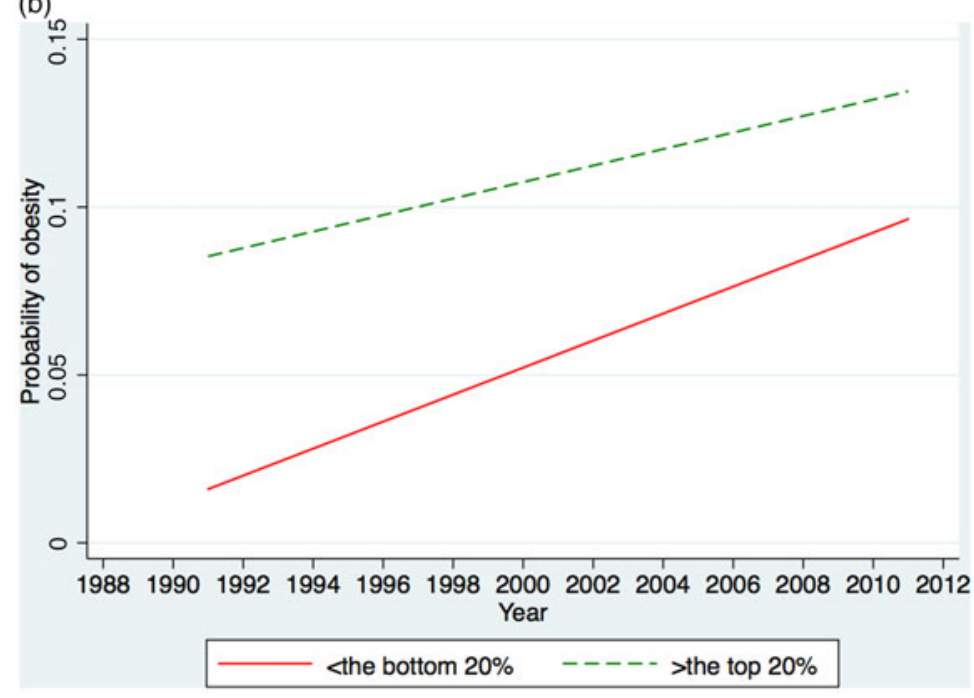

Figure 1. Trends in the influence of urbanisation on the probabilities of obesity among males (a) and females (b) aged $\geqslant 45$ years in China.

may suggest that older people, especially women from the less urbanised/rural areas, may be more vulnerable to China's urbanisation process in terms of hypertension risk. Results of the time trends of Hukou, a rural-urban fixed measure of urbanisation, on CVD risks showed similar patterns and are reported in the online supplementary material. However, the use of the urbanisation index enabled us to explore the full spectrum of urbanisation levels and its association with time trends of CVD risks. 
(a)

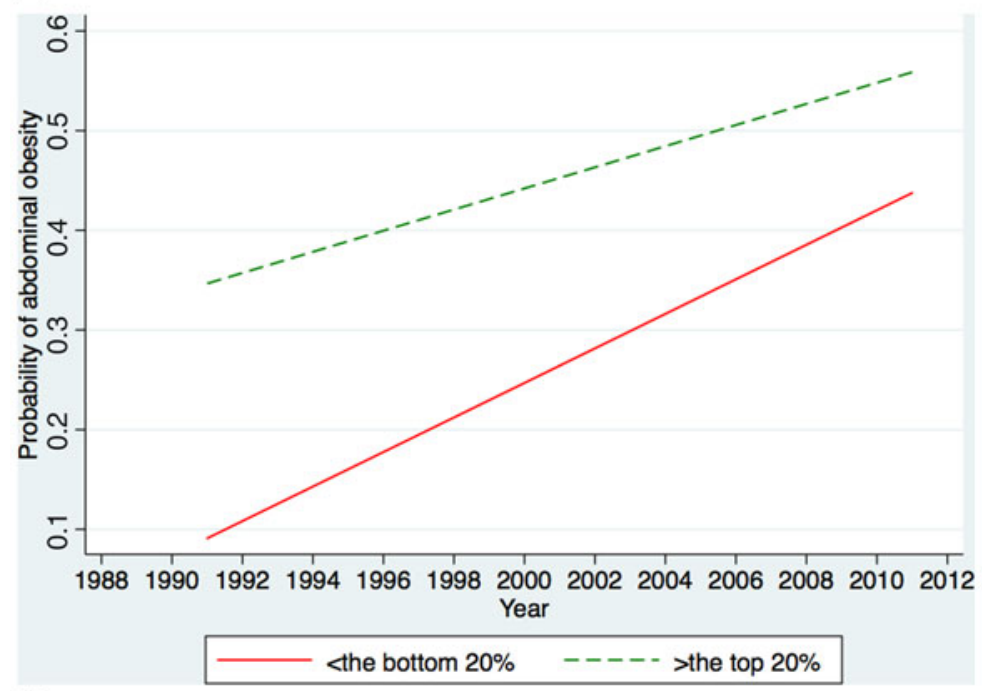

(b)

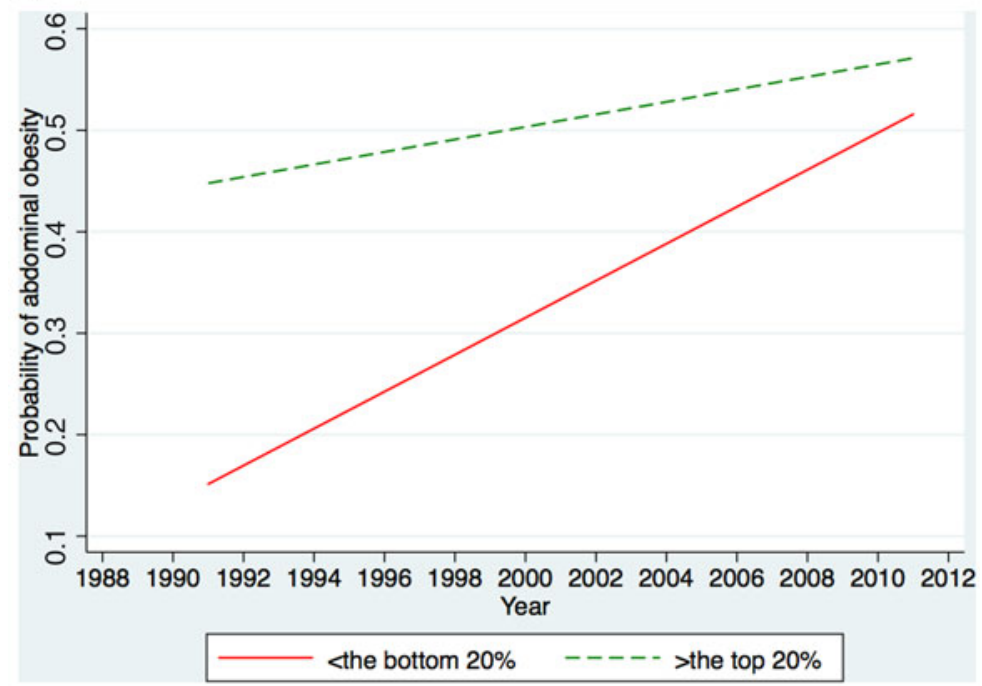

Figure 2. Trends in the influence of urbanisation on the probabilities of abdominal obesity among males (a) and females (b) aged $\geqslant 45$ years in China.

\section{Discussion}

This study has drawn on a nationwide longitudinal data-set - the CHNS - to examine time trends in related CVD risks among middle-aged and older people aged $\geqslant 45$ years old in China by considering an important wider contextual factor urbanisation. Chinese middle-aged and older people have been experiencing an upward trend in CVD risks in the last two decades. An upward trend in CVD risks (except for blood pressure) was observed over two decades of urbanisation, 
(a)

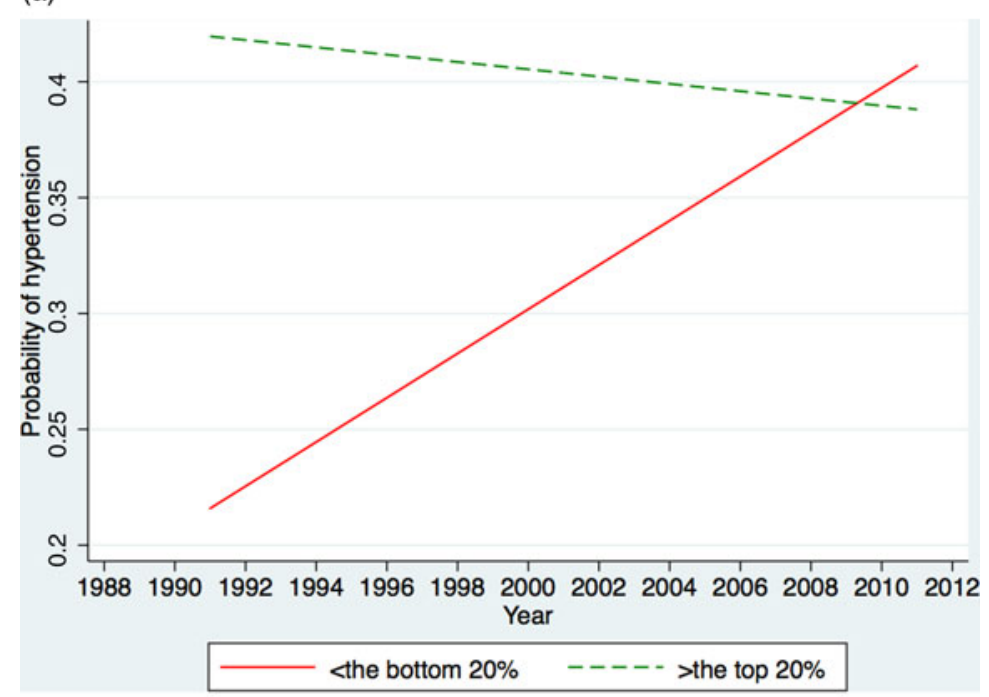

(b)

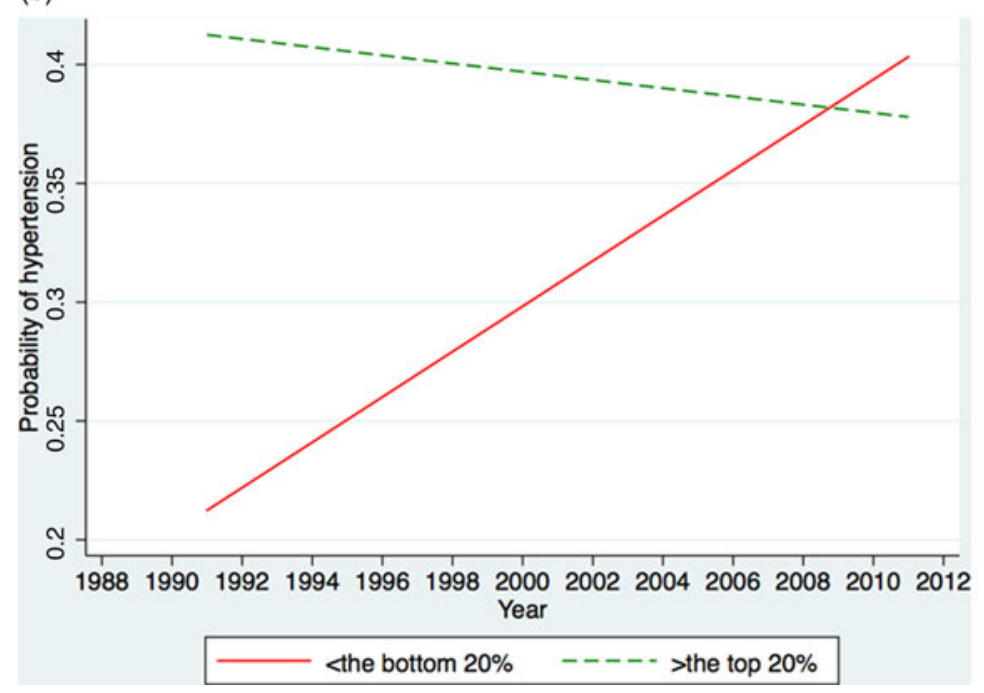

Figure 3. Trends in the influence of urbanisation on the probabilities of hypertension among males (a) and females (b) aged $\geqslant 45$ years in China.

especially among women. However, urbanisation may reduce inequality among women between more urbanised areas and less urbanised areas. This study has contributed to current debates on rural-urban inequalities in the health and wellbeing of older people in China at least in two ways. First, the use of multi-level modelling techniques in this study allows disentangling of the effects due to individual, family and neighbourhood characteristics, which is methodologically more robust. Another advantage of this study is, unlike previous studies mostly drawing on 
the crude classification of the rural-urban dichotomy which is prone to misclassification error, the urbanisation measure used has encompassed dimensions beyond population size and density. It has captured some important dimensions of social, economic and physical characteristics of urban living over time and space (Zhang et al., 2014).

After adjustment for relevant confounders at the individual and household levels, positive time trends in all CVD risks (BMI, WC, SBP and DBP) among middle-aged and older people over two decades were found. The prevalence of obesity and hypertension in general increases constantly over time from 1991 to 2011. This is consistent with previous studies that found an increased prevalence of obesity and hypertension among elderly Chinese people (Yu et al., 2000; Gordon-Larsen et al., 2014; Wang et al., 2014).

This study suggested that urbanisation has contributed to an increased risk of CVD among the middle-aged and elderly in China. It was found that urban residents tend to have higher a prevalence of obesity and hypertension than their rural counterparts. The gap shrinks over time, especially for women. Urban residents in China tend to have higher obesity rates than rural residents due to the potentially negative influence of urban living regarding unhealthy diets and lifestyles (physical inactivity and increased access to high-fat and energy-dense diets), as well as exposure to obesogenic environments (Popkin, 1999; Zhai et al., 2014; Li et al., 2016). Westernised diets are associated with an increased likelihood of obesity, hypertension and the metabolic syndrome (Sun et al., 2014). In recent years, the consumption of a traditional diet has decreased and that of high-energy food has increased substantially at low- and middle-income levels, which has shifted the risk of obesity from high-income groups to middle- and low-income groups and poor and rural areas (Dearth-Wesley et al., 2007). Moreover, those from rural and/or less-urbanised areas tend to have less favourable health services compared to those from urban or more urbanised areas, including access to medical facilities, the availability of medical insurance and the quality of health services (Hou, 2008). This may cause under-diagnosis and under-treatment of hypertension (Hou, 2008; Song et al., 2014), which may contribute to rural residents catching up with urban residents in terms of CVD risks. It is important to examine potential contributing factors such as lifestyles and health-care services more closely, which may explain rural-urban disparities in CVD of older people in China.

Substantial gender differences in CVD risks among middle-aged and older people in China were observed, despite indirect comparisons. In general women tend to have a higher prevalence of abdominal obesity than men, which is consistent with a previous study (Song et al., 2018). One possible explanation might be because although men were more likely to adopt an unhealthy lifestyle such as smoking and drinking, women were more likely to be physically inactive than men at older age (Song et al., 2014). In general, women from the rural or less-urbanised areas appear to be worse off in CVD risks over two decades of urbanisation than rural males (Wang et al., 2014). Rural women tend to live longer and are more likely to be widowed than rural men (Zeng and George, 2010). Massive internal migration (mostly taken up by the working-age population) and rapid urbanisation are likely to drive the ageing problem to be more challenging in rural than in urban China (Chen et al., 2015). Due to lack of institutional social 
welfare and support, they are more likely to rely on their families compared to their urban peers. However, rapid urbanisation and migration have enhanced the geographical separation between adult children and their elderly parents, restricting familial social support (Joseph and Phillips, 1999; Chen and Liu, 2009). This is likely to have significant implications for CVD profiles of older women from the less-urbanised communities in China.

Figures 1-3 also suggest that over two decades, the gap between women from the rural or least urbanised areas and those from the urban or most urbanised areas narrow much more rapidly than their male counterparts. This may suggest that the urbanisation process may help reduce gender inequality in CVD risks over time. One possible explanation is that urbanisation is associated with improved economic circumstances and better access to health-care services ( $\mathrm{Li}$ and $\mathrm{Wu}, 2016$ ), whereas men tend to be less likely to use health-care services than their female counterparts (Galdas et al., 2005). This may persist as people age. Therefore, it is possible that, in China, men at older age may not take advantage of urbanisation as much as women. Previous studies that have examined this among older people in China have not been found. Future studies are needed to further elucidate pathways through which urbanisation contributes to reducing gender inequality in China.

\section{Limitations}

Several methodological limitations warrant cautious interpretation of the findings. The first concerns the measure of urbanisation. Urbanisation is a complex phenomenon that involves social, economic and environmental factors that are exogenous to the individual (Vlahov et al., 2005; Smith and Rappaport, 2009). Although the classification of urbanisation used has captured some important dimensions of social, economic and physical characteristics of urban living over time and space (Zhang et al., 2014), there are still other unobserved facets in relation to the urban environment that matter to older people's health and wellbeing, such as pollution (Baumgartner et al., 2011). For example, environmental risk factors, in particular air and water pollution, are one of the major contributors to morbidity and mortality in China (Li et al., 2016). Future studies may benefit by collecting a wide array of data on different dimensions of urbanisation in order to disentangle causal links between urbanisation and outcomes. Also, some confounders such as unhealthy diets and lifestyles as mediating factors that operate from urbanisation to CVD risk were not included in this study because disentangling pathways is beyond the focus of this study. Moreover, one key limitation in the CHNS, as in any longitudinal data-set, is missing data and sample attrition. This study only included complete cases and this may lead to biased results.

The different mechanisms through which urbanisation affects CVD risks among middle-aged and older people in China could not be distinguished. The pathways can be complex and multi-factorial. Freudenberg et al. (2005) proposed an alternative and comprehensive approach that should incorporate and integrate the penalty and sprawl concepts as well as consider other features of living in cities that influence health. Dimensions of urban living conditions include the physical environment, the social environment, health and social services systems, and the characteristics of urban populations (i.e. behaviours, beliefs and demographics) 
(Freudenberg et al., 2005). Future studies may benefit by further exploring and identifying modifiable risk factors and pathways that can prevent avoidable health inequalities.

One important pathway is through improved household economic circumstances. A growing body of research has examined the changing nature of income or wealth inequality in China during its urbanisation in the last few decades. It is hypothesised that during the process of urbanisation, while more people move from the lowerincome rural sector to the higher-income urban sector, the overall income inequality will first increase then decrease, following an inverse U-shaped curve (Kuznets, 1955). China is believed to have already passed the turning point, therefore urbanisation should help reduce income inequality (Kanbur, 2014). Urbanisation in China has significantly reduced both poverty of rural residents and migrant workers and, consequently, has contributed to narrowing down the rural-urban income gap. It may, however, contribute to urban poverty due to the competition between migrant workers and urban workers in urban labour markets and the failure of the government's anti-poverty policies in urban settings (Zhang, 2016). Despite an immediate alleviating effect on income inequality generally during post-reform, urbanisation in China seems to have a lagged aggravating effect on income inequality whereas neglecting the social dimension of urbanisation may further cause a longterm economic and social segregation such as the rural-urban gap and the persistent marginalisation of rural migrants (Chen et al., 2016). Future studies are needed to further distinguish heterogeneities among social groups of older people and their distinctive socio-economic circumstances.

\section{Conclusion}

This study provides an overall picture of time trends of CVD risks among Chinese middle-aged and older people and how it changes with urbanisation over the last two decades. The rapid growth of the ageing population will pose tremendous challenges to the public health and social welfare system in China. The findings may provide a sound evidence basis to inform decision-making processes in relation to tackling health and welfare inequalities among older people in China by targeting the most vulnerable groups. There is a possibility for policy intervention to reduce inequality during the process of China's planned urbanisation.

Supplementary material. The supplementary material for this article can be found at https://doi.org/10. 1017/S0144686X18001794.

Data. This research uses data from China Health and Nutrition Survey (CHNS). The National Institute for Nutrition and Health, China Center for Disease Control and Prevention, Carolina Population Center (P2C HD050924, T32 HD007168), the University of North Carolina at Chapel Hill, the National Institutes of Health (NIH) (R01-HD30880, DK056350, R24 HD050924, R01-HD38700) and the NIH Fogarty International Center (D43 TW009077, D43 TW007709) supported the CHNS data collection and analysis files from 1989 to 2015 and future surveys. The China-Japan Friendship Hospital, Ministry of Health supported CHNS 2009, the Chinese National Human Genome Center at Shanghai has supported the CHNS since 2009 and the Beijing Municipal Center for Disease Prevention and Control has supported the CHNS since 2011.

Author ORCIDs. (D) Nan Zhang, 0000-0002-8032-275X. 
Financial support. This work was supported by the UK Economic and Social Research Council Global Challenge Research Fund Fellowship (grant number ES/P009824/1) and a Hallsworth Research Fellowship.

Conflict of interest. The author declares no conflicts of interest.

Ethical standards. The institutional review committees from the University of North Carolina at Chapel Hill and the National Institute for Nutrition and Food Safety, China Centre for Disease Control and Prevention approved the survey protocols and instruments and the process for obtaining informed consent for the survey.

\section{References}

Baumgartner J, Schauer JJ, Ezzati M, Lu L, Cheng C, Patz JA and Bautista LE (2011) Indoor air pollution and blood pressure in adult women living in rural China. Environmental Health Perspectives 119, $1390-1395$.

Blumenthal S, Epps R, Heavenrich R, Lauer R, Lieberman E, Mirkin B, Mitchell S, Boyar NV, O'Hare D and McFate SW (1977) Report of the task force on blood pressure control in children. Pediatrics 59, 797-820.

Cai F and Wang D (2005) Demographic transition: implications for growth. In Garnaut R and Song L (eds), The China Boom and its Discontents. Canberra: Asia Pacific Press, 34-52.

Chan KW and Buckingham W (2008) Is China abolishing the hukou system? The China Quarterly 195, 582-605.

Chatterji S, Kowal P, Mathers C, Naidoo N, Verdes E, Smith JP and Suzman R (2008) The health of aging populations in China and India. Health Affairs 27, 1052-1063.

Chen F and Liu G (2009) Population ageing in China. In Uhlenberg P (ed.), International Handbook of Population Aging. New York, NY: Springer, 157-172.

Chen G, Glasmeier AK, Zhang M and Shao Y (2016) Urbanization and income inequality in post-reform China: a causal analysis based on time series data. PLOS ONE 11, e0158826.

Chen Q, Dietzenbacher E and Los B (2015) The Effects of Ageing and Urbanization on China's Future Population and Labor Force. Groningen, The Netherlands: University of Groningen, Research Institute SOM: Systems, Organisations and Management.

Dahly DL and Adair LS (2007) Quantifying the urban environment: a scale measure of urbanicity outperforms the urban-rural dichotomy. Social Science \& Medicine 64, 1407-1419.

Dearth-Wesley T, Wang H and Popkin B (2007) Obesity dynamics in China: the poor are catching up. European Journal of Clinical Nutrition 18, 1-6.

Freudenberg N, Galea S and Vlahov D (2005) Beyond urban penalty and urban sprawl: back to living conditions as the focus of urban health. Journal of Community Health 30, 1-11.

Galdas PM, Cheater F and Marshall P (2005) Men and health help-seeking behaviour: literature review. Journal of Advanced Nursing 49, 616-623.

Godfrey R and Julien M (2005) Urbanisation and health. Clinical Medicine 5, 137-141.

Gordon-Larsen P, Wang H and Popkin BM (2014) Overweight dynamics in Chinese children and adults. Obesity Reviews 15, 37-48.

Gu D, Reynolds K, Wu X, Chen J, Duan X, Muntner P, Huang G, Reynolds R, Su S, Whelton P and He J (2002) The International Collaborative Study of Cardiovascular Disease in ASIA. Prevalence, awareness, treatment, and control of hypertension in China. Hypertension 40, 920-927.

Hou X (2008) Urban-rural disparity of overweight, hypertension, undiagnosed hypertension, and untreated hypertension in China. Asia Pacific Journal of Public Health 20, 159-169.

Hugo G (2017) New Forms of Urbanization: Beyond the Urban-Rural Dichotomy. London: Routledge.

Jones-Smith JC and Popkin BM (2010) Understanding community context and adult health changes in China: development of an urbanicity scale. Social Science \& Medicine 71, 1436-1446.

Joseph AE and Phillips DR (1999) Ageing in rural China: impacts of increasing diversity in family and community resources. Journal of Cross-cultural Gerontology 14, 153-168.

Kanbur R (2014) Inequality in Asia and the Pacific: Trends, Drivers, and Policy Implications. London: Routledge.

Kuznets S (1955) Economic growth and income inequality. American Economic Review 45, 1-28. 
Li M and Wu Y (2016) Urbanization and Public Health in China. London: Imperial College Press.

Li X, Song J, Lin T, Dixon J, Zhang G and Ye H (2016) Urbanization and health in China, thinking at the national, local and individual levels. Environmental Health 15, 114-123.

Ma LJ (2002) Urban transformation in China, 1949-2000: a review and research agenda. Environment and Planning A 34, 1545-1570.

McDade TW and Adair LS (2001) Defining the 'urban' in urbanization and health: a factor analysis approach. Social Science \& Medicine 53, 55-70.

McGee T (2008) Managing the rural-urban transformation in East Asia in the 21st century. Sustainability Science 3, 155-167.

Moore M, Gould P and Keary BS (2003) Global urbanization and impact on health. International Journal of Hygiene and Environmental Health 206, 269-278.

Moran A, Gu D, Zhao D, Coxson P, Wang YC, Chen C-S, Liu J, Cheng J, Bibbins-Domingo K and Shen Y-M (2010) Future cardiovascular disease in China: Markov model and risk factor scenario projections from the coronary heart disease policy model - China. Circulation: Cardiovascular Quality and Outcomes 3, 243-252.

National Bureau of Statistics of China (2015) China Statistical Yearbook 2015. Beijing: China Statistics Press. Available at http://www.stats.gov.cn/tjsj/ndsj/2015/indexeh.htm.

Popkin BM (1999) Urbanization, lifestyle changes and the nutrition transition. World Development 27, 1905-1916.

Popkin BM, Du S, Zhai F and Zhang B (2009) Cohort profile: the China Health and Nutrition Survey-monitoring and understanding socio-economic and health change in China, 1989-2011. International Journal of Epidemiology 39, 1435-1440.

Smith MT and Rappaport SM (2009) Building exposure biology centers to put the E into 'G $\times$ E' interaction studies. Environmental Health Perspectives 117, A334-A335.

Song A, Liang Y, Yan Z, Sun B, Cai C, Jiang H and Qiu C (2014) Highly prevalent and poorly controlled cardiovascular risk factors among Chinese elderly people living in the rural community. European Journal of Preventive Cardiology 21, 1267-1274.

Song MN, X, C, JX, K and Wang HM (2018) Prevalence and influencing factors of overweight obesity among middle-aged and elderly people in China. Chinese Journal of Disease Control and Prevention 22, 804-808.

Statacorp (2013) Stata Statistical Software: Release 13. College Station, TX: StataCorp LP.

Sun J, Buys NJ and Hills AP (2014) Dietary pattern and its association with the prevalence of obesity, hypertension and other cardiovascular risk factors among Chinese older adults. International Journal of Environmental Research and Public Health 11, 3956-3971.

Tuan NT, Adair LS, Stevens J and Popkin BM (2010) Prediction of hypertension by different anthropometric indices in adults: the change in estimate approach. Public Health Nutrition 13, 639-646.

United Nations Department of Economic and Social Affairs (2015) World Population Prospects: The 2015 Revision. New York, NY: United Nations Department of Economic and Social Affairs, Population Division. Available at https://esa.un.org/unpd/wpp/.

Vlahov D, Galea S, Gibble E and Freudenberg N (2005) Perspectives on urban conditions and population health. Cadernos de Saúde Pública 21, 949-957.

Wang J, Ning X, Yang L, Lu H, Tu J, Jin W, Zhang W and Su T (2014) Trends of hypertension prevalence, awareness, treatment and control in rural areas of northern China during 1991-2011. Journal of Human Hypertension 28, 25-31.

World Health Organization (1997) Obesity: Preventing and Managing the Global Epidemic. Report of a WHO Consultation on Obesity. Geneva: World Health Organization.

Yu Z, Nissinen A, Vartiainen E, Song G, Guo Z and Tian H (2000) Changes in cardiovascular risk factors in different socioeconomic groups: seven year trends in a Chinese urban population. Journal of Epidemiology \& Community Health 54, 692-696.

Zeng $\mathbf{Y}$ and George $\mathbf{L}$ (2010) Population ageing and old-age insurance in China. In Dannefer D and Phillipson C (eds), The Sage Handbook of Social Gerontology. London: Sage, 420-429.

Zhai F, Du S, Wang Z, Zhang J, Du W and Popkin B (2014) Dynamics of the Chinese diet and the role of urbanicity, 1991-2011. Obesity Reviews 15, 16-26.

Zhang B, Zhai F, Du S and Popkin BM (2014) The China Health and Nutrition Survey, 1989-2011. Obesity Reviews 15, 2-7. 
Zhang Y (2016) Urbanization, inequality, and poverty in the People's Republic of China. Asian Development Bank Institute, Tokyo, ADBI Working Paper.

Zhang $\mathbf{Z}$ and Treiman DJ (2013) Social origins, hukou conversion, and the wellbeing of urban residents in contemporary China. Social Science Research 42, 71-89.

Zhou B (2002) Predictive values of body mass index and waist circumference for risk factors of certain related diseases in Chinese adults - study on optimal cut-off points of body mass index and waist circumference in Chinese adults. Biomedical and Environmental Sciences 15, 83-96.

Zhu Y, Ioannidis JP, Li H, Jones KC and Martin FL (2011) Understanding and Harnessing the Health Effects of Rapid Urbanization in China. Environmental Science \& Technology 45, 5099-5104.

Cite this article: Zhang N (2020). Urban-rural disparities in cardiovascular disease risks among middleaged and older Chinese: two decades of urbanisation. Ageing \& Society 40, 1405-1427. https://doi.org/ $10.1017 /$ S0144686X18001794 